\begin{tabular}{|l|l|l|}
\hline \multicolumn{2}{|c|}{ PublisherInfo } \\
\hline \hline PublisherName & $:$ & BioMed Central \\
\hline \hline PublisherLocation & $:$ & London \\
\hline \hline PublisherImprintName & $:$ & BioMed Central \\
\hline \hline
\end{tabular}

\title{
GH treatment for the critically ill
}

\begin{tabular}{|l|l|l||}
\hline \multicolumn{2}{|c||}{ ArticleInfo } \\
\hline \hline ArticleID & $:$ & 4102 \\
\hline \hline ArticleDOI & $:$ & $10.1186 /$ ccf-1999-2021 \\
\hline \hline ArticleCitationID & $:$ & 2021 \\
\hline \hline ArticleSequenceNumber & $:$ & 39 \\
\hline \hline ArticleCategory & $:$ & Paper Report \\
\hline ArticleFirstPage & $:$ & 1 \\
\hline \hline ArticleLastPage & $:$ & 4 \\
\hline \hline & & RegistrationDate : 1999-10-1 \\
ArticleHistory & $:$ & OnlineDate \\
\hline \hline ArticleCopyright & $:$ & Current Science Ltd1999-10-1 \\
\hline \hline ArticleGrants & $:$ & \\
\hline \hline ArticleContext & $:$ & 130541111 \\
\hline \hline
\end{tabular}




\section{Keywords}

Critically ill, growth hormone, outcome

\section{Comments}

The twofold increase in mortality seen with high dose GH treatment in the critically ill contrasts with results of previous studies in predominantly burns and trauma patients where, at the worst, there was no difference compared to placebo. The mechanisms to explain this mortality are probably multifactorial and the timing and dose of GH treatment undoubtedly have an important influence on how the inflammatory processes are modulated. Previously we have seen that high doses of steroids have detrimental effects in the critically ill, but we are now seeing a resurgence in interest of steroid treatment in the ICU since it appears that physiological doses have beneficial effects. No doubt GH treatment in this population will be revisited when the timing and dose of treatment required to have favourable effects on the inflammatory response are better understood.

Finally, it is a pity that the first interim analysis was never reached before the trial design was changed. Presumably the study would have been stopped at an earlier stage because the excess mortality was so high.

\section{Introduction}

There is evidence that the increased catabolic response to injury is partly a result of resistance to growth hormone (GH) and to reduced circulating insulin-like growth factor (IGF-1). Consequently, it was proposed that administration of GH in the critically ill would attenuate this response, resulting in less respiratory muscle weakness and so reduced requirements for mechanical ventilation and therefore shorter ICU stays. Although it has previously been shown (especially in burns and trauma patients) that GH does improve nitrogen balance as a result of a reduced catabolic response, there is little evidence that this translates into fewer days on ventilation, reduced ICU stays or improved outcome. Two large multicenter studies were designed to answer these questions.

\section{Aims}


To determine whether GH, when administered to critically ill patients, results in a better outcome and a reduction in ventilator, ICU, and hospital days.

\section{Methods}

Two prospective, European, multicenter, double-blind, randomized, placebo-controlled studies running in parallel involved a total of 532 patients. Patients expected to have prolonged ICU stays and who had already been in the ICU for 5-7 days were recruited. Exclusions included septic shock, diabetes, liver failure and burns. Patients were randomized to receive high doses of GH (5-20 times usual replacement doses) or placebo and this continued for a maximum of 21 days. Nitrogen intake was controlled, assessments of muscle strength/fatigue were performed, and IGF-1 and IGF-binding proteins were assayed. Although originally designed as group sequential trials, this was changed to a fixedsample analysis before the first interim analysis was made.

\section{Results}

Patients receiving GH had twice the mortality (39\% and 44\%) compared with the placebo groups (20\% and 18\%), with the main causes of death being septic shock and multiple-organ failure (MOF). The time course for the increased mortality differed between the two groups, with the majority occurring within the first 10 days of treatment in one study, but was more evenly distributed over the first 30 days in the other. Durations of ventilation, ICU and hospital stay were longer in those survivors who received $\mathrm{GH}$, and there appeared to be no differences in muscle strength/fatigue between the two groups. Serum IGF-1 concentrations increased to a greater extent and hyperglycemia was recorded more frequently as an adverse event in the $\mathrm{GH}$ groups.

\section{Discussion}

Treatment of critically ill patients with GH has a strongly negative effect on outcome and even though there was an improvement in nitrogen balance in these patients, this did not translate into improvements in muscle strength. In fact duration of ventilation, ICU and hospital stays was longer in the GH treated groups. The fact that the majority of deaths were a consequence of septic shock or MOF makes it likely that GH in this situation has an adverse effect on immune function (in the laboratory GH can promote or inhibit production of various inflammatory mediators, depending on the experimental conditions). Hyperglcemia is known to increase the risk of sepsis and this was seen more often in the GH groups as a metabolic consequence of this hormone. Another factor may be a reduced availability of glutamine for leukocytes and enterocytes as a consequence of GH treatment. Previous studies have shown no deleterious effects on outcome with GH treatment in the septic patient, and so the mechanisms to 
explain the negative findings in this multicenter study are probably more complex than given above. They may also depend on when GH is given in the course of the illness and the dose given.

\section{Additional information}

An accompanying editorial tackles the mechanism behind the increased mortality seen with GH treatment in this patient population, contrasting with the more favorable effects on outcome seen in patients with severe trauma or burns. GH was given at the peak of the inflammatory response in the studies involving burns and trauma patients, whereas in this study the inflammatory response was yet to start and so the timing of treatment may have a powerful influence on whether beneficial or deleterious effects are seen.

\section{References}

1. Takala J, Ruokonen E: Increased mortality associated with growth hormone treatment in critically ill adults. N Engl J Med. 1999, 341: 785-792. 